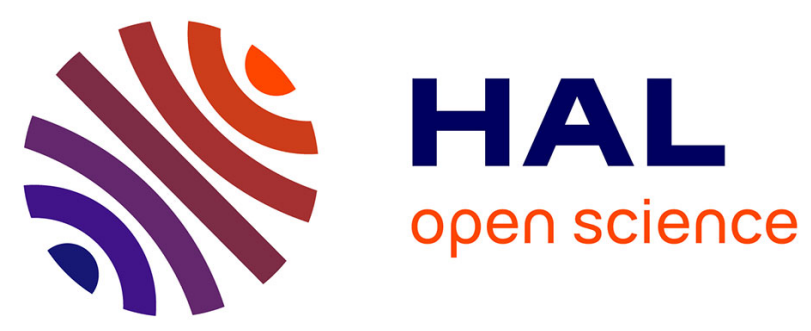

\title{
Investigation of the electrical limit conditions effects in piezoelectric transducer arrays utilized in medical imaging
}

Imane Laasri, Abdelmajid Bybi, Ouadia Mouhat, Mohammad Jamal, Anne-Christine Hladky, Aziz Ettahir, Kamal Kettani

\section{To cite this version:}

Imane Laasri, Abdelmajid Bybi, Ouadia Mouhat, Mohammad Jamal, Anne-Christine Hladky, et al.. Investigation of the electrical limit conditions effects in piezoelectric transducer arrays utilized in medical imaging. Applied Acoustics, 2020, 10.1016/j.apacoust.2020.107509 . hal-03019835

\section{HAL Id: hal-03019835 \\ https://hal.science/hal-03019835}

Submitted on 7 Dec 2020

HAL is a multi-disciplinary open access archive for the deposit and dissemination of scientific research documents, whether they are published or not. The documents may come from teaching and research institutions in France or abroad, or from public or private research centers.
L'archive ouverte pluridisciplinaire HAL, est destinée au dépôt et à la diffusion de documents scientifiques de niveau recherche, publiés ou non, émanant des établissements d'enseignement et de recherche français ou étrangers, des laboratoires publics ou privés. 
Imane LAASRI ${ }^{1}$, Abdelmajid BYBI ${ }^{2 *}$, Ouadia MOUHAT ${ }^{3}$, Mohammad JAMAL ${ }^{1}$, Anne Christine HLADKY ${ }^{4}$, Aziz ETTAHIR ${ }^{2}$, and Kamal KETTANI ${ }^{2}$

\section{${ }^{1}$ Hassan II University of Casablanca, Faculty of Sciences Ben M'sik, Department of Physics,} Engineering and Materials Laboratory (LIMAT), Engineering and Mechanics Team.

${ }^{2,3}$ Mohammed V University in Rabat, Ecole Supérieure de Technologie de Salé

${ }^{2}$ MEAT - Materials Energy and Acoustics Team

${ }^{3}$ LGCE - Laboratoire Génie Civil et Environnement

${ }^{4}$ Univ. Lille, CNRS, Centrale Lille, ISEN, UPHF, UMR 8520, IEMN Lille, France

* Corresponding author: abdelmajid.bybi@um5.ac.ma

Ecole Supérieure de Technologie de Salé

Avenue Le Prince Héritier, B.P 227, 11000 Salé Médina. Maroc

\section{Abstract}

Crosstalk is considered as an undesirable phenomenon disturbing the electromechanical behavior of the ultrasonic transducer arrays used in medical imaging applications. Indeed, when one element of a transducer array is excited, it generates parasitic voltages and/or displacement fields on the adjacent passive elements. Consequently, these interactions between elements decrease the array's electroacoustic performance, which affects the obtained image quality. To overcome the crosstalk's problem, several research works propose active cancellation techniques. In this case, the correction voltages are determined by considering the array's elements grounded, contrary to the conventional crosstalk's definition which considers the array elements in Open-Circuit. The novelty of this paper is in one hand the study of the electrical limit conditions' effects on the physical behavior of a piezoelectric transducer array radiating in a fluid medium (water). On the other hand, a displacement method is proposed to evaluate the crosstalk level when the array elements are grounded. The limitations of the proposed method are also discussed. For this purpose, a piezoelectric transducer array is firstly modeled using a Two-dimensional Finite Elements Method (FEM), when the array elements are not grounded (open-circuit). Then, the results are compared to those obtained when the neighboring elements are grounded (as in the case of the crosstalk's active cancellation techniques). 
Finally, measurements are realized on a fabricated transducer array vibrating in air medium and are successfully compared to the results predicted using FEM.

Keywords-Piezoelectric transducer arrays, displacement measurement, electrical limit conditions.

\section{Introduction}

Ultrasonic transducers and transducer arrays are usually utilized in medical diagnostics and therapeutic applications [1-5]. The major objective of ongoing and future research in this area is to optimize the electroacoustic performance of these transducers, to obtain a high image resolution for more reliable and safe diagnostics. For this purpose, several works investigate the crosstalk phenomenon, which decreases the performance of the ultrasonic transducer arrays [6-14]. Indeed, when one element of a transducer array is driven, it generates parasitic voltages and/or displacement fields on the passive neighboring elements, which affects the performance of these devices. It is mainly responsible for anomalous behavior in the directivity of the ultrasonic transducer arrays, i.e. in presence of crosstalk, the main lobe is not obtained in the axial direction of the transducer array and several undesirable side lobes can be observed [1516]. The crosstalk level in the ultrasonic transducer arrays is generally evaluated as the ratio between the parasitic voltages obtained on the passive neighboring elements and the excitation's amplitude applied to the active element [11, 14]. In this definition, one element of the studied transducer array is electrically driven, while its neighboring elements are considered electrically in Open-Circuit (not grounded).

In literature research works devoted to the minimization of crosstalk can be divided into three approaches. The first one investigates the contribution of the passive elements, i.e. filling material, matching layers, and backing, to the mechanical crosstalk [17-21]. The second approach consists of developing a systematic method for the active cancellation of crosstalk. The last method concerns specific treatments realized on the excitation and reception signals to reduce crosstalk [22-23]. The active cancellation of crosstalk is based on the application of adequate voltages to the elements adjacent to the excited one, to minimize the parasitic signals (crosstalk). The required voltages can be determined using different methods. Cugnet et al. [24] proposed a numerical technique to calculate them from the average normal displacement computed at the surface of the array elements. In the same manner, Bybi et al. [16] determined the correction voltages from punctual displacement measurements realized using a Laser Vibrometer. Zhou et al. [25-26] developed another 
method using the transfer function matrix relating input voltages $V i$ to output pressures $P i$. In this case, the measurement of the elements transfer function is realized using a hydrophone. Finally, Bybi et al. [7, 27] proposed another simple electrical method to cancel crosstalk in acoustical arrays, using the analogy between the motional current and normal displacement. In this situation, the determination of the correction voltages requires average measurements, i.e. impedance and current measurements, instead of punctual displacement measurements.

In all active crosstalk cancellation techniques, the determination of the correction voltages considers the neighboring elements electrically in Short-Circuit, i.e. the passive neighboring elements are connected to the ground, contrary to the conventional definition (neighboring elements are not grounded). The main objective of this paper is to study the effects of the electrical limit conditions (neighboring elements grounded or not grounded) on the electromechanical behavior of the piezoelectric transducer arrays utilized in medical imaging. An alternative solution to the conventional crosstalk's evaluation method, i.e. based on the ratio between the parasitic voltages measured on each passive element and the excitation voltage, is also proposed and tested when the array elements are grounded. It is expected that this study will be helpful in terms of crosstalk definition and accurate evaluation and suppression.

The first part of this paper is devoted to the description of a conventional piezoelectric transducer array and the presentation of the experimental setup utilized to evaluate the crosstalk level. In the second part, a transducer array composed of seven piezoelectric elements made of PZ 27 ceramic is modeled using a two-dimensional finite element method. In the last section, a prototype is fabricated and the experimental results, i.e. electrical impedance and displacement curves are compared to the numerical ones (FEM). Finally, the crosstalk level is evaluated experimentally considering both electrical limit conditions: neighboring elements ground and not grounded.

\section{Piezoelectric transducer arrays for medical imaging applications}

\subsection{Structure description}

Transducer arrays utilized in medical imaging and NDT applications are generally composed of an even number of piezoelectric elements having a thickness $T$, a width $W$, and a length $L$, spaced by a distance $d$ and aligned as illustrated in Fig. 1. The elements are polarized in the thickness direction (z-direction) and are bonded to each other by a nonconductive resin. The transducer array elements' are also equipped with front and back 
matching layers, to minimize the mismatching problem, which creates a prolonged ringing after pulse excitation. Generally, the acoustic impedance of a conventional transducer is matched to that of the propagation medium (tissues in medical applications) by one or two matching layers on its front face and a thick backing layer on its back face. In our study, the manufactured array is only composed of piezoelectric elements bonded to each other by a non-conductive acrylic resin, PLEXCIL (ESCIL). The matching layers are not taken into account to facilitate the fabrication of the prototype. This kind of transducer arrays is utilized to understand the crosstalk phenomenon and to study the effects of the electrical limit conditions on the array's electromechanical behavior. This also allowed as testing our crosstalk correction methods easily [16, 27].

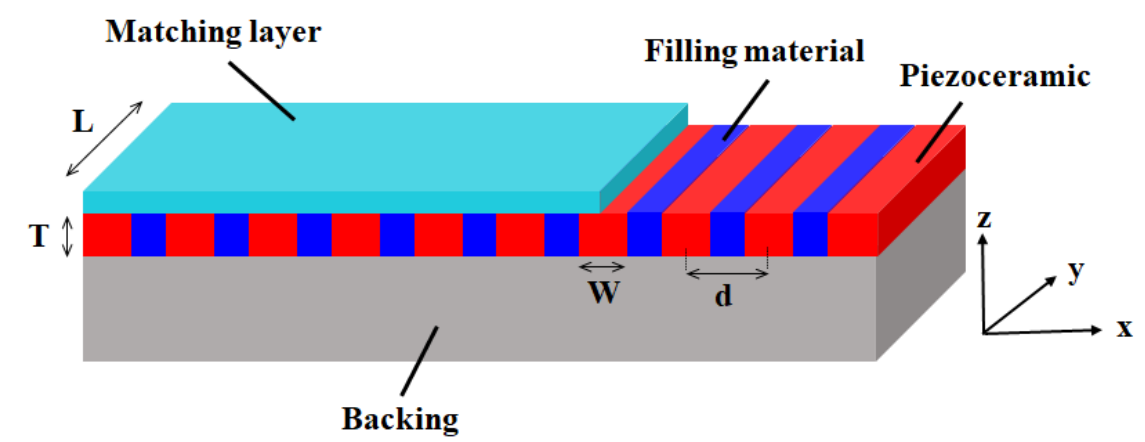

Figure 1: Schematic description of a conventional piezoelectric transducer array.

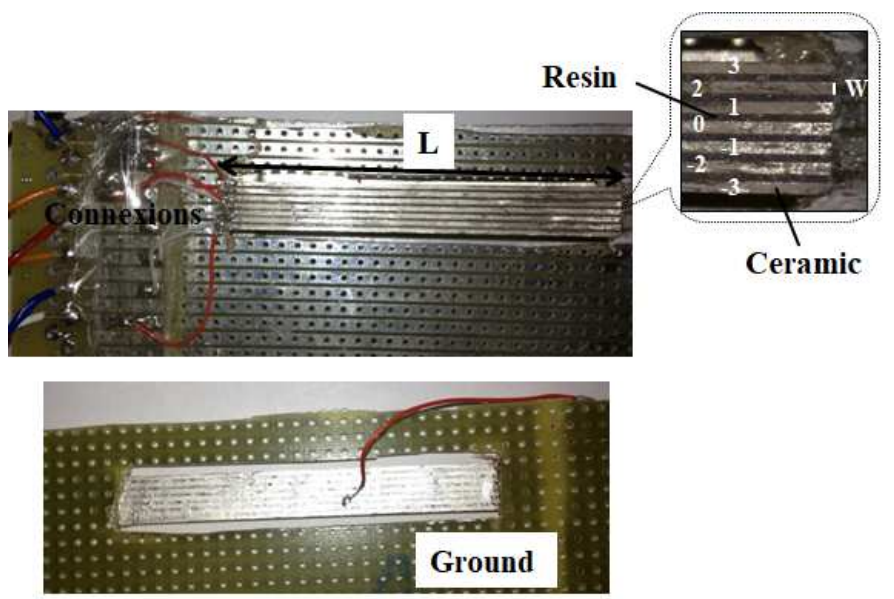

Figure 2: Seven-element transducer array.

To get a full symmetry of the transducer array and to simplify calculations and analysis, an odd number of transducers is assumed. As shown in Fig. 2, the fabricated prototype is composed of seven piezoelectric elements made of PZ27 Ferroperm ceramic, having the following dimensions: $T=3.3 \mathrm{~mm}, W=0.7 \mathrm{~mm}, L=37 \mathrm{~mm}$ and $d=1.2 \mathrm{~mm}$. The piezoelectric material (PZ27) properties are listed in Table I. 


\begin{tabular}{|c|c|c|c|c|c|c|c|c|c|c|c|}
\hline $\begin{array}{c}S_{11}^{E} \\
\mathrm{pPa}^{-1}\end{array}$ & $\begin{array}{c}S_{12}^{E} \\
\mathrm{pPa}^{-1}\end{array}$ & $\begin{array}{c}S_{13}^{E} \\
\mathrm{pPa}^{-1}\end{array}$ & $\begin{array}{c}S_{33}^{E} \\
\mathrm{pPa}^{-1}\end{array}$ & $\begin{array}{c}S_{44}^{E} \\
\mathrm{pPa}^{-1}\end{array}$ & $\begin{array}{c}S_{66}^{E} \\
\mathrm{pPa}^{-1}\end{array}$ & $\begin{array}{c}d_{15} \\
\mathrm{pC} / \mathrm{N}\end{array}$ & $\begin{array}{c}d_{31} \\
\mathrm{pC} / \mathrm{N}\end{array}$ & $\begin{array}{c}d_{33} \\
\mathrm{pC} / \mathrm{N}\end{array}$ & $\frac{\varepsilon_{33}^{S}}{\varepsilon_{0}}$ & $\frac{\varepsilon_{11}^{S}}{\varepsilon_{0}}$ & $\begin{array}{c}\rho \\
\mathrm{kg} / \mathrm{m}^{3}\end{array}$ \\
\hline 17 & -6.71 & -8.53 & 23 & 43.47 & 47.42 & 500 & -170 & 425 & 1130 & 914 & 7700 \\
\hline
\end{tabular}

Table I: Physical properties of PZ27 ceramic.

\subsection{Crosstalk measurements}

Crosstalk measurements are performed on the studied transducer array using two methods as shown in Fig 3 and Fig. 4. In the first technique (Fig. 3), the central element "0" is excited using Agilent 33250A Low Frequency Generator delivering an electrical sine signal $V_{0}$. The neighboring elements "1", "-1", "2", "-2", "3" and "-3" are connected individually to a digital oscilloscope, displaying the parasitic voltage generated on each element $\left(V_{i}, i=1,-1,2,-\right.$ $2,3$, and -3$)$. In the literature $[11,14]$ the crosstalk level $C(\mathrm{~dB})$ is evaluated from the measurements using the relation (1):

$$
C(\mathrm{~dB})=20 \log \left(\frac{V_{i}}{V_{0}}\right)
$$

This definition considers one element of the studied transducer array as electrically driven, while its neighboring elements are electrically in Open-Circuit (not grounded). In this paper, a second method is proposed to evaluate the crosstalk level when the neighboring elements are connected to the ground, as in the case of the crosstalk's active cancellation methods [16, 24, $25,26,27]$. In this situation, voltage measurement cannot be utilized because the elements are not in Open-Circuit as suggested by the definition (Fig.3).

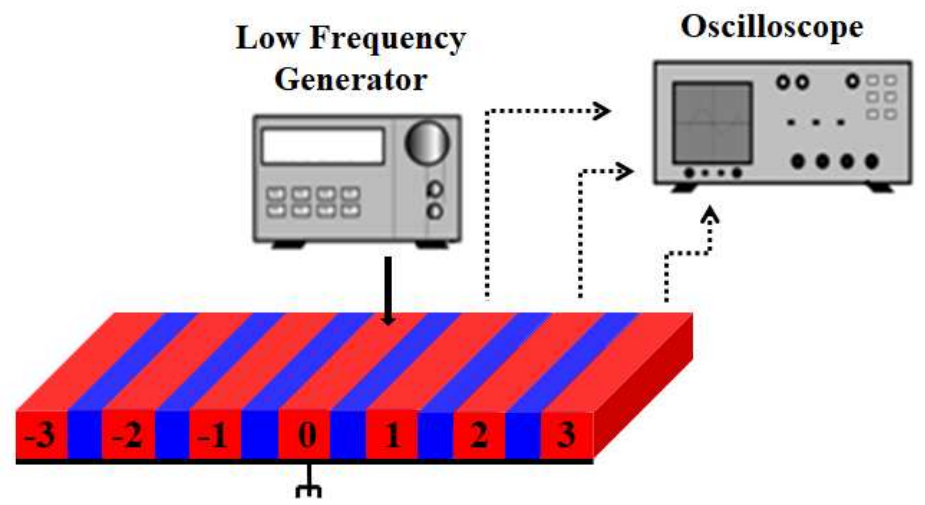

Figure 3: Crosstalk's evaluation using voltage measurements. 
Another solution is to utilize the displacement measurements instead of the voltage ones as shown in Fig. 4. One of the objectives of this research work is to test the validity of this method and to evaluate its limitations. For this purpose, displacement measurements are carried out in the middle of the fabricated array elements using a Polytech psv400 laser vibrometer (Fig. 4). The results obtained using both methods (Fig. 3 and 4) are compared numerically and experimentally in the frequency domain.

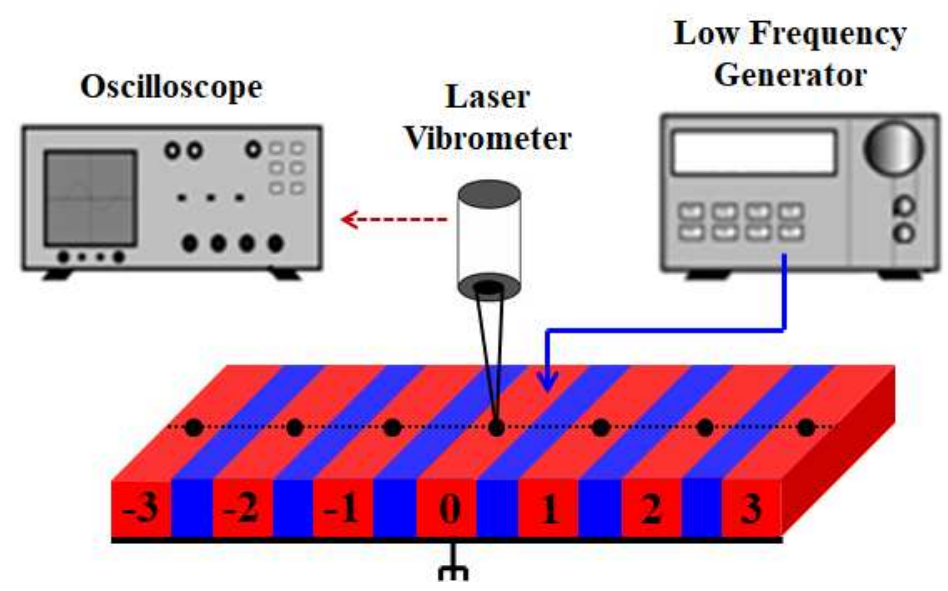

Figure 4: Crosstalk’s evaluation using displacement measurements.

\section{Numerical study}

The piezoelectric transducer array is first modeled numerically using the ATILA code (FEM code for elastic and piezoelectric structures radiating in fluid) [28] to determine its Transmitting Voltage Response (TVR) and to predict the normal displacement at the radiating surface of each array element. The numerical model is also utilized to compute the array's electrical impedance and to evaluate the crosstalk level. As shown in Fig. 5(a), the studied array is composed of seven piezoelectric elements made of PZ27 ceramic bounded to each other by Acrylic PLEXCIL resin (filling material). Both the piezoelectric array elements and the filling material (resin) are meshed using isoparametric quadratic elements respecting the $\lambda / 4$ criterion, i.e. the piezoelectric array elements are divided into seven elements along their width $W$ and fifty elements along their thickness $T$, whereas the resin elements are divided into five elements along their width and fifty elements along their thickness. The spacing between the array elements $(d)$ is chosen equal to $1.2 \mathrm{~mm}$ to respect the Nyquist criterion and to avoid grating lobes. Each element of the studied array has a thickness $T=3.3 \mathrm{~mm}$, a width $W=0.7$ $\mathrm{mm}$ and its length is chosen to be $37 \mathrm{~mm}$ long, with a Length-to-Thickness Ratio $(L / T)$ of about 11.21, thus this length can be considered as infinite and a plane strain approximation 
assumed. In the literature, it is demonstrated that a dominant thickness mode is obtained when the width-to-thickness ratio $\mathrm{W} / \mathrm{T}$ is less than or equal to 0.5 [29-30]. This aspect ratio is chosen about 0.21 to enable the separation of the thickness mode about the undesirable lateral modes (width and length modes). Furthermore, in our previous work (ref. 8), it was also demonstrated numerically and experimentally that the influence and the contribution of these undesirable modes (width and length modes) is negligible. The transducer array radiates in a fluid medium (water in medical imaging applications) meshed with isoparametric quadratic elements respecting the $\lambda / 4$ criterion. This medium is limited by a non-reflecting surface $\Gamma e$ made up of dipolar elements that absorb the outgoing acoustic wave almost completely [15]. Furthermore, only half the domain is meshed due to symmetry.

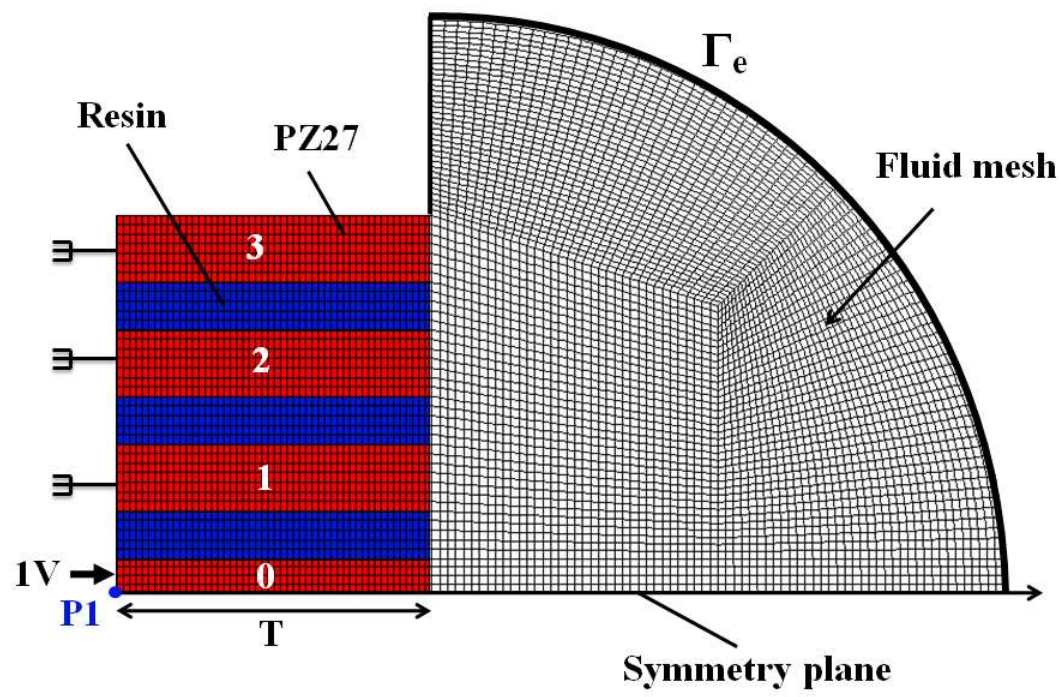

(a) Structure's mesh.

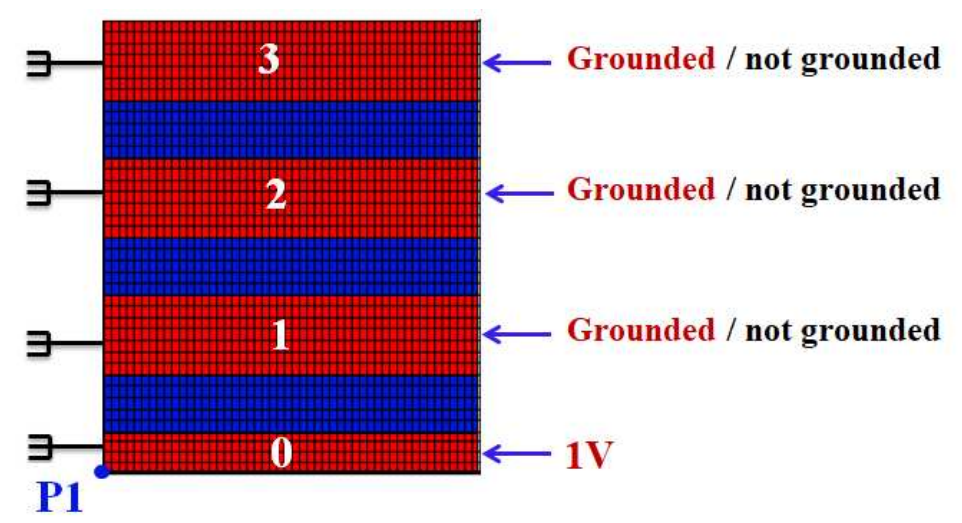

(b) Electrical limit conditions.

Figure 5: Schematic description of a seven-element transducer array radiating in water. 
To study the effects of the electrical limit conditions on the array's electromechanical behavior, two limit conditions are considered (Fig. 5(b)):

i) The array's central element "0" is driven, while its neighboring elements " 1 ", "2" and "3" are grounded.

ii) The central element " 0 " is driven and its neighboring elements "1", "2" and " 3 " are not grounded (open-circuit).

\subsection{Transmitting Voltage Response (TVR)}

Firstly, the Transmitting Voltage Response (TVR) of the seven-element transducer array is computed between $250 \mathrm{kHz}$ and $750 \mathrm{kHz}$, for a relative reference pressure $1 \mu \mathrm{Pa} / \mathrm{V}$ at $1 \mathrm{~m}$. Fig. 6 compares the TVR obtained considering the electrical limit conditions (i) and (ii). After analysis, it is observed that the two curves follow relatively the same variation, i.e. the same maximums and minimums. Nevertheless, a frequency shift about $20 \mathrm{kHz}$ is obtained between the curves. Furthermore, a parasitic mode (maximum of TVR) is observed around the frequency of $570 \mathrm{kHz}$, in the curve corresponding to the limit condition (ii). Finally, the maximum of TVR obtained for the limit condition (i) is somewhat less than that computed for the condition (ii) (about $2 \mathrm{~dB}$ ).

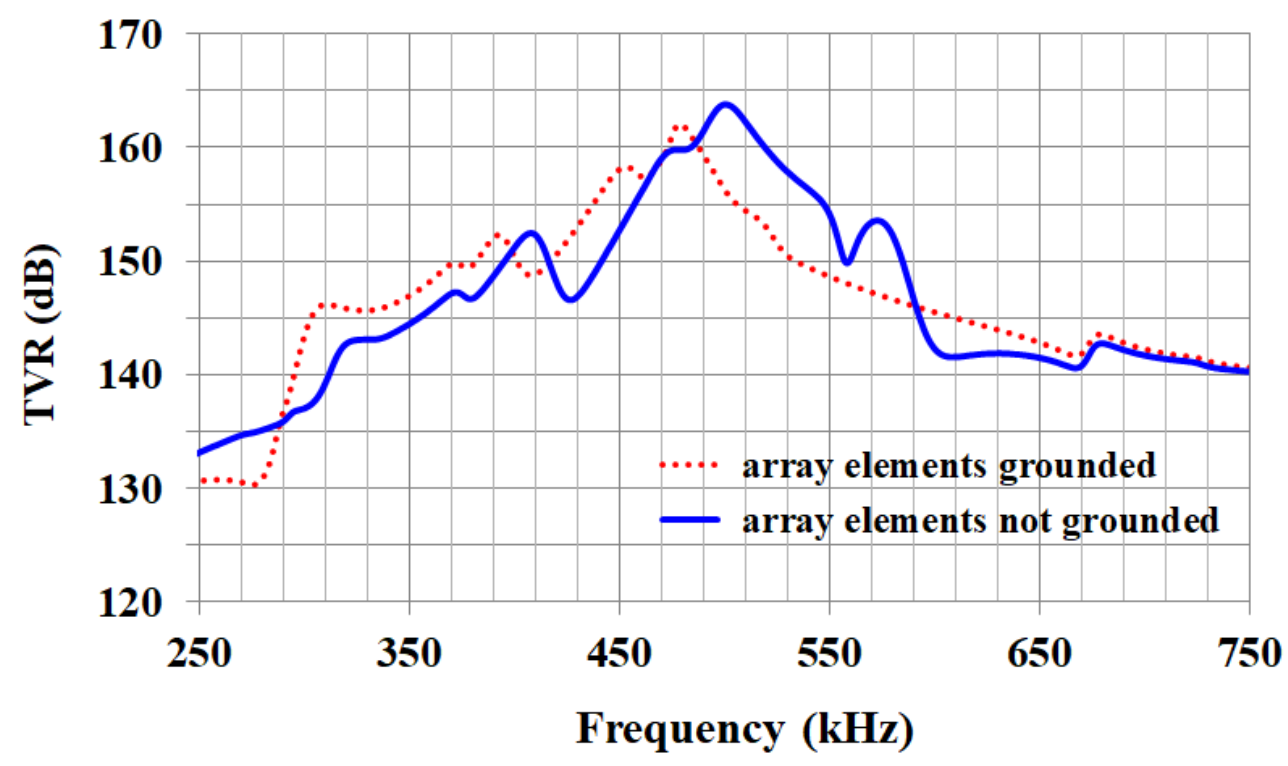

Figure 6: Transmitting Voltage Response (TVR) of the seven-element transducer array.

\subsection{Displacement}

The normal displacement is also computed in the middle of the radiating surface of the central element "0", i.e. at the position P1 (Fig. 5b) between $250 \mathrm{kHz}$ and $750 \mathrm{kHz}$. Fig. 7 
compares the normalized displacement curves (all values are divided by the maximum value reached by one of the compared curves) obtained considering the electrical limit conditions (i) and (ii). It is observed from this figure that the results are different, particularly between 450 $\mathrm{kHz}$ and $550 \mathrm{kHz}$. In this frequency domain, a maximum of displacement is obtained around $500 \mathrm{kHz}$ (mechanical resonant frequency), for the limit condition (ii). Whereas, in the case of the limit condition (i), two resonance frequencies $475 \mathrm{kHz}$ and $520 \mathrm{kHz}$ are obtained. Finally, the comparison of the two curves indicates a frequency shift (about $25 \mathrm{kHz}$ ) between the two displacement peaks observed in the frequency band $450 \mathrm{kHz}-550 \mathrm{kHz}$.

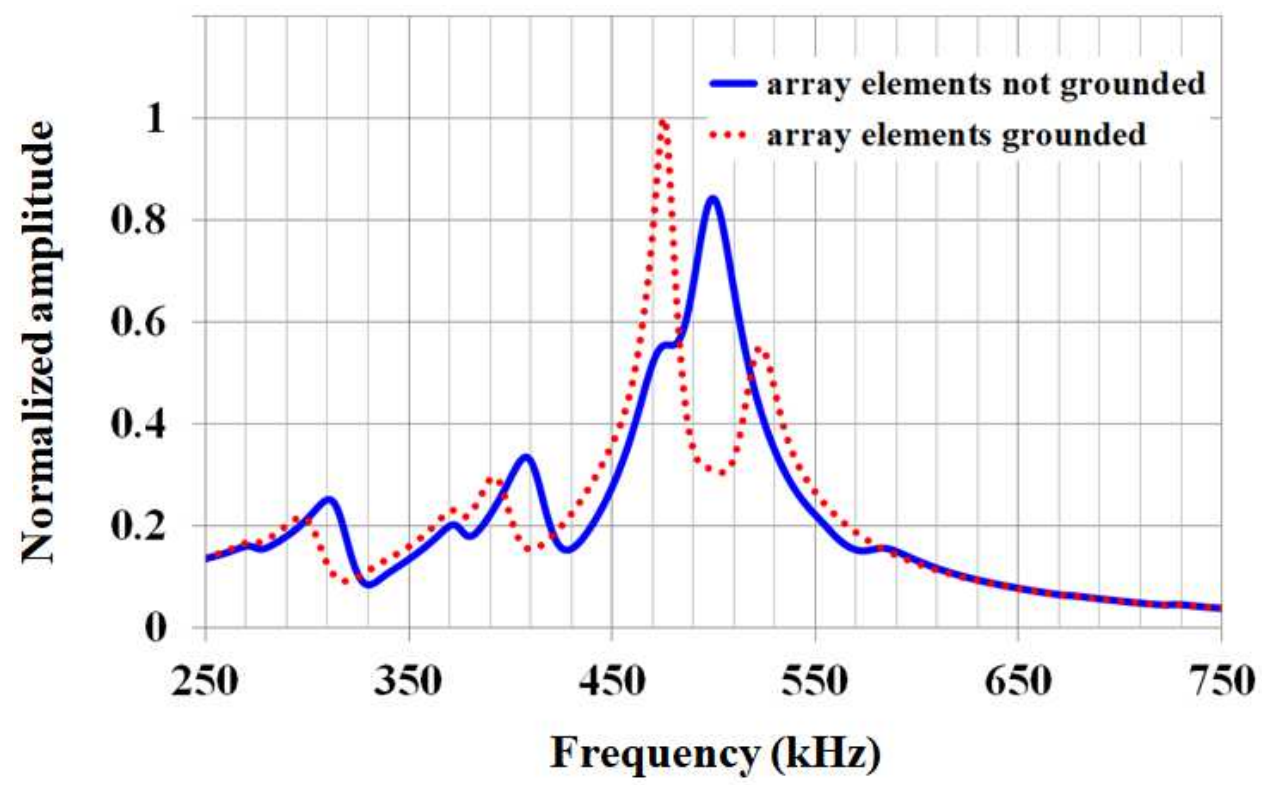

Figure 7: Normal displacement computed at the radiating surface of the element "0".

\subsection{Electrical impedance}

To observe the effect of the limit conditions (i) and (ii) on the electrical behavior of the transducer array, the electrical impedances computed using the numerical model are compared in the frequency domain $250 \mathrm{kHz}-750 \mathrm{kHz}$, as shown in Fig. 8. After analysis, it is observed that the two curves follow the same behavior in the frequency domains $250 \mathrm{kHz}-$ $470 \mathrm{kHz}$ and $590 \mathrm{kHz}-750 \mathrm{kHz}$. Nevertheless, a frequency shift (about $20 \mathrm{kHz}$ ) is obtained between the two curves in the first frequency band. The major differences are observed around the thickness mode, i.e. in the frequency band $470 \mathrm{kHz}-590 \mathrm{kHz}$. In this domain, two resonance frequencies $(475 \mathrm{kHz}$ and $520 \mathrm{kHz})$ and a one anti-resonance frequency $(581 \mathrm{kHz})$ are obtained for the limit condition (i), whereas in the case of the condition (ii), one resonant frequency is obtained at about $500 \mathrm{kHz}$ and two anti-resonance frequencies are observed at $571 \mathrm{kHz}$ and $595 \mathrm{kHz}$. As seen previously in Fig. 7, the resonance frequencies (minimum of 

displacement).

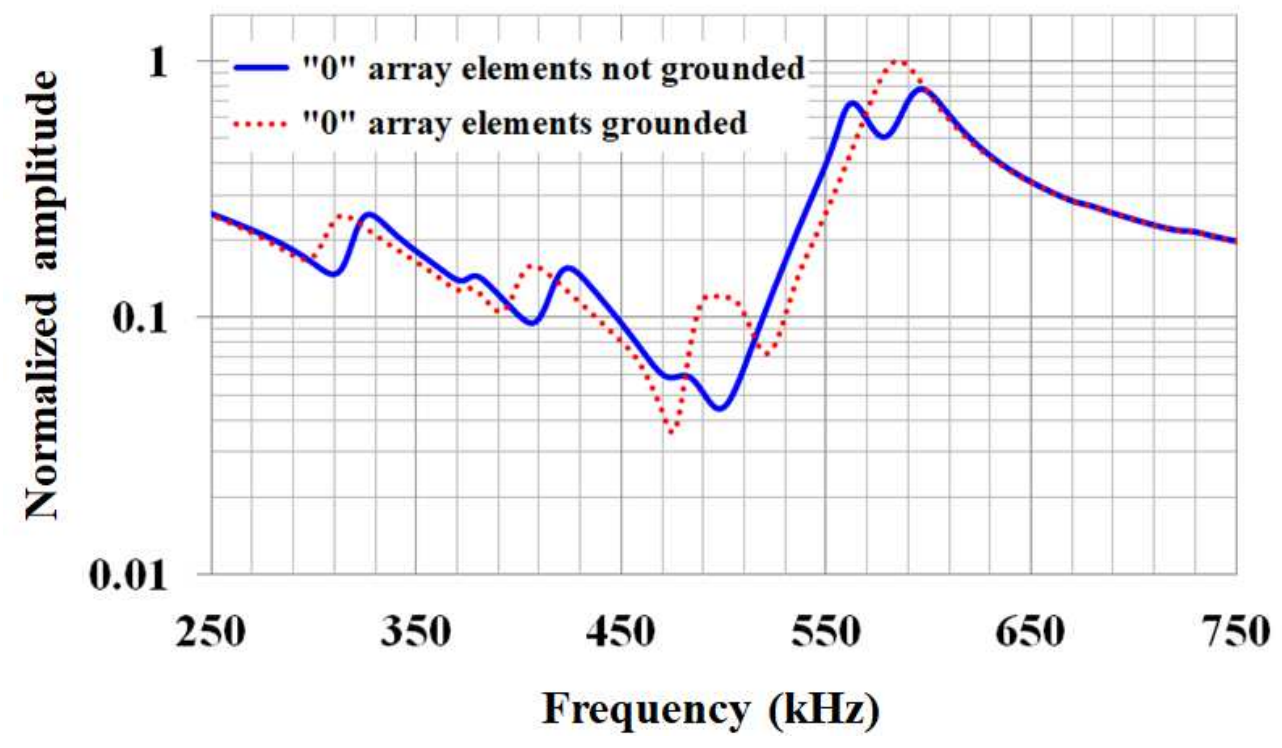

Figure 8: Numerical electrical impedance of the central element " 0 ".

The differences between the results obtained under the limit conditions (i) and (ii), i.e. TVR, displacement and impedance curves, can be explained by the simplified equivalent circuit represented in Fig. 9. In this case, only two piezoelectric elements bonded to each other by a non-conductive resin are considered, i.e. an active piezoelectric element (part 1) and its neighbouring element grounded or in open-circuit (part 2). The piezoelectric elements are represented by their equivalent electromechanical circuit composed of an electrical part $\left(R_{01,2}, C_{01,2}\right)$ and a mechanical part $\left(R_{1,2}, L_{1,2}\right.$ and $\left.C_{1,2}\right)$. The elements $R_{1,2}, L_{1,2}$ and $C_{1,2}$ correspond to a mass - spring system, for which $R_{1,2}$ represents the mechanical dissipations, $L_{1,2}$ the mass and $C_{1,2}$ the compliance (flexibility) of the material. $R_{01,2}$ and $C_{01,2}$ represent the dielectric losses and the static capacitance of the piezoelectric material. The two parts are connected by a transformer converting the electrical energy to mechanical energy and viceversa and having a transformation ratio $N$. Because of their very large value (several $\mathrm{M} \Omega$ ), the resistors $R_{01,2}$ are neglected. In the same manner, the non-conductive resin is represented by its equivalent circuit $\left(L_{R}, C_{R}\right)$. In our previous work [7], it was demonstrated that this representation is more accurate in the vicinity of the considered resonant frequency (thickness resonance). According to the equivalent circuit, it is clear that when the element 2 is grounded, its static capacitance $C_{02}$ is short-circuited, i.e. the contribution of the electrical branch $\left(C_{02}\right)$ is suppressed. Consequently, only the mechanical branch $\left(R_{2}, L_{2}\right.$ and $\left.C_{2}\right)$ contributes to the total electrical impedance of the structure composed of the elements 1 and 
2. Finally, in the case of the transducer array composed of seven piezoelectric elements, the limit condition (i) is responsible in the elimination of the individual static capacitances and it results in non-negligible differences between the curves obtained under the limit conditions (i) and (ii), e.g. the observed frequency shifts.

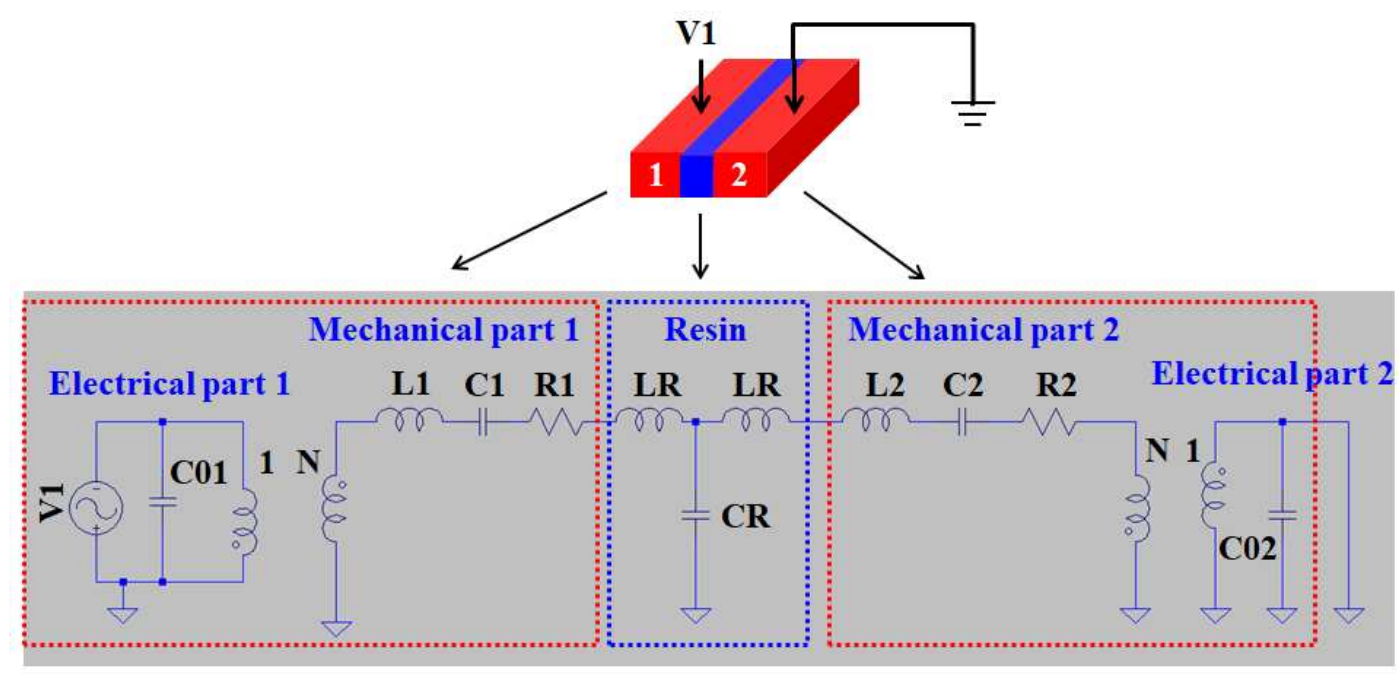

Figure 9: Equivalent circuit of two piezoelectric elements bonded to each other by resin.

\subsection{Crosstalk}

The crosstalk level is firstly evaluated at the two characteristic frequencies (maximum of displacement) $475 \mathrm{kHz}$ and $520 \mathrm{kHz}$ using both methods. The estimation of the crosstalk level $C$ utilizes the conventional method (relation (1)) when the neighboring elements are not grounded (limit condition (ii)). In the case of the limit condition (i) (neighboring elements grounded) the proposed method is to evaluate the crosstalk from the displacement values in the middle of the array elements) using the relation (2):

$$
C(\mathrm{~dB})=20 \log \left(\frac{u_{i}}{u_{0}}\right)
$$

where $u_{0}$ represents the normal displacement computed in the middle of the central element " 0 " and $u_{i}(i=1,2,3)$ the displacement in the middle of the neighboring elements "1", "2" and "3". The results obtained using the two methods are summarized in Table II. The comparison shows that the two methods, i.e. evaluation of crosstalk level by the relation (1) and using parasitic displacements instead of voltages $C_{u}$ (relation 2), give relatively similar crosstalk values at the resonance frequencies. The difference (about $1 \mathrm{~dB}$ ) is probably because the definition of the crosstalk is based on the voltage, which is an average value, whereas, the displacement's method is punctual (obtained in the middle of the elements). 


\begin{tabular}{|c|c|c|c|}
\hline Element & “1” & “2” & “3” \\
\hline \multirow{2}{*}{ Crosstalk $C_{\boldsymbol{u}}(\mathbf{d B})$} & -11.8 & -5.2 & -6.1 \\
\cline { 2 - 4 } & -8.8 & -7.9 & -10 \\
\hline & -12.7 & -7.4 & -7.7 \\
\hline \multirow{2}{*}{ Crosstalk $C(\mathbf{d B})$} & -8.1 & -6.7 & -11.1 \\
\cline { 2 - 4 } & & & \\
\hline
\end{tabular}

Table II: Crosstalk evaluated at the resonance frequencies $475 \mathrm{kHz}$ and $520 \mathrm{kHz}$ (bold values).

The comparison is then extended to a large frequency band between $250 \mathrm{kHz}$ and 650 $\mathrm{kHz}$, as shown in Fig. 10. After analysis, it is observed that the crosstalk evaluated using both methods are similar around the two characteristic frequencies (see dashed rectangular). The difference between the two curves increases beyond the resonance frequencies. In other words, the crosstalk evaluated using the displacement in the middle of the array elements is a good approximation in the vicinity of the considered mode (thickness mode in this work).

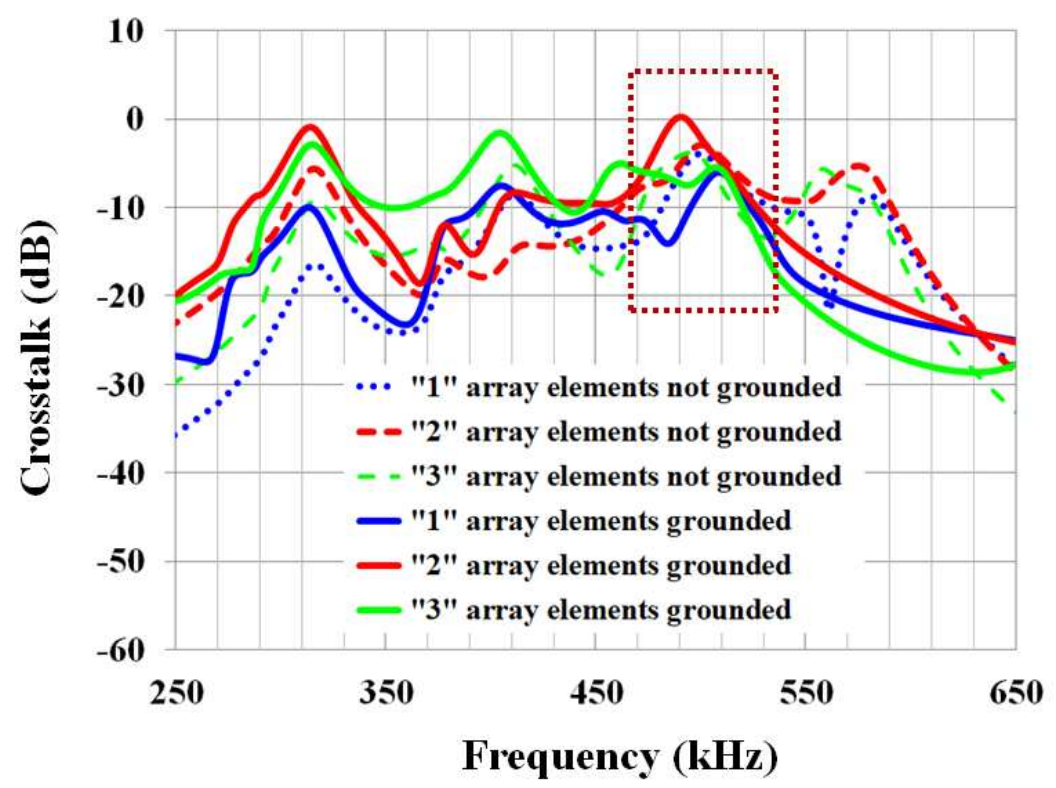

Figure 10: Crosstalk computed using relation 1 (array elements not grounded) compared to that obtained from the relation 2 (array elements grounded).

\section{Experimental Characterization}

The manufactured transducer array (Fig. 2), is characterized experimentally by electrical impedance and displacement measurements, in both conditions (i) and (ii). The crosstalk level 
is also evaluated using the previous methods, i.e. from the parasitic voltages (relation (1)) and using the proposed displacements method (relation (2)). For the sake of simplification, measurements are realized in air. The results are then compared to those obtained numerically in the air medium instead of water. It is important to notice that the FEM calculations are first done in the water medium (section 3) to see the effects of the limit conditions on the transducer arrays utilized in medical imaging, and then done in the air medium to compare the numerical results with the experimental ones.

\subsection{Displacement}

To avoid the edge effects, displacement measurements are realized in the middle of the central element "0" using a Polytech OFV353 Laser Vibrometer. The curves obtained in both conditions (i) and (ii) are compared with that measured in the middle of a single element (Fig. 11).

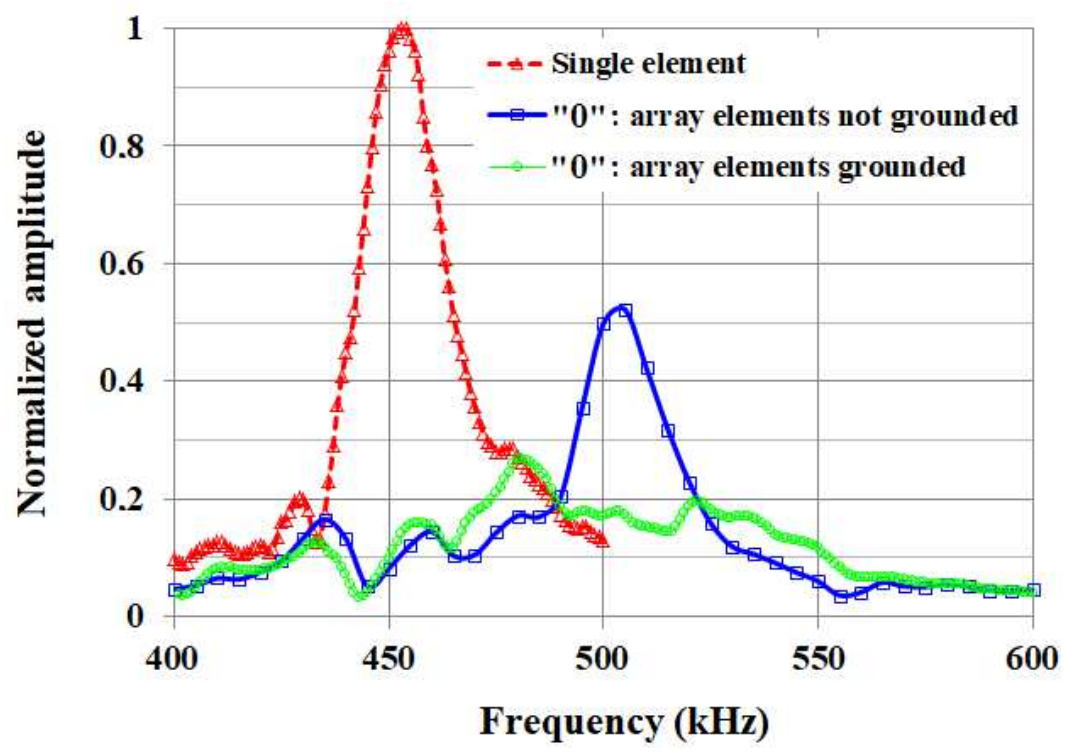

Figure 11: Normalized displacement amplitude measured in the middle of the central element " 0 " compared to that obtained in the middle of a single piezoelectric element.

As expected, the curves measured on the element "0" (conditions (i) and (ii)) are different, particularly in the frequency band $450 \mathrm{kHz}-550 \mathrm{kHz}$. In this domain, the maximum displacement is obtained at about $500 \mathrm{kHz}$ for the condition (ii). Whereas, in the case of the condition (i), two mechanical resonance frequencies $481 \mathrm{kHz}$ and $520 \mathrm{kHz}$ are obtained. Furthermore, the displacement amplitudes at both frequencies are lower than those observed at $500 \mathrm{kHz}$. Finally, a frequency shift is obtained between the single element's resonance frequency $(452 \mathrm{kHz})$ and the array's resonance frequencies. This result is due to the 
presence of the neighboring elements, which shifts the resonance frequencies towards the high frequencies and reduces the amplitude of the displacement, e.g. the maximum displacement for the condition (ii) is about half that obtained in the case of the single element.

Fig. 12 compares the displacements measured in the middle of the element " 1 ", in both conditions (i) and (ii) to the numerical results (FEM). It is seen from this figure that the results obtained are similar. Nevertheless, a small frequency shift is observed between the curves (about $4 \mathrm{kHz}$ ), due to the materials' incertitude. Furthermore, the displacement's amplitude obtained numerically is relatively high compared to the measured one, because the resin's real losses are not taken into account (about 5\%) and the piezoelectric material losses are not considered.

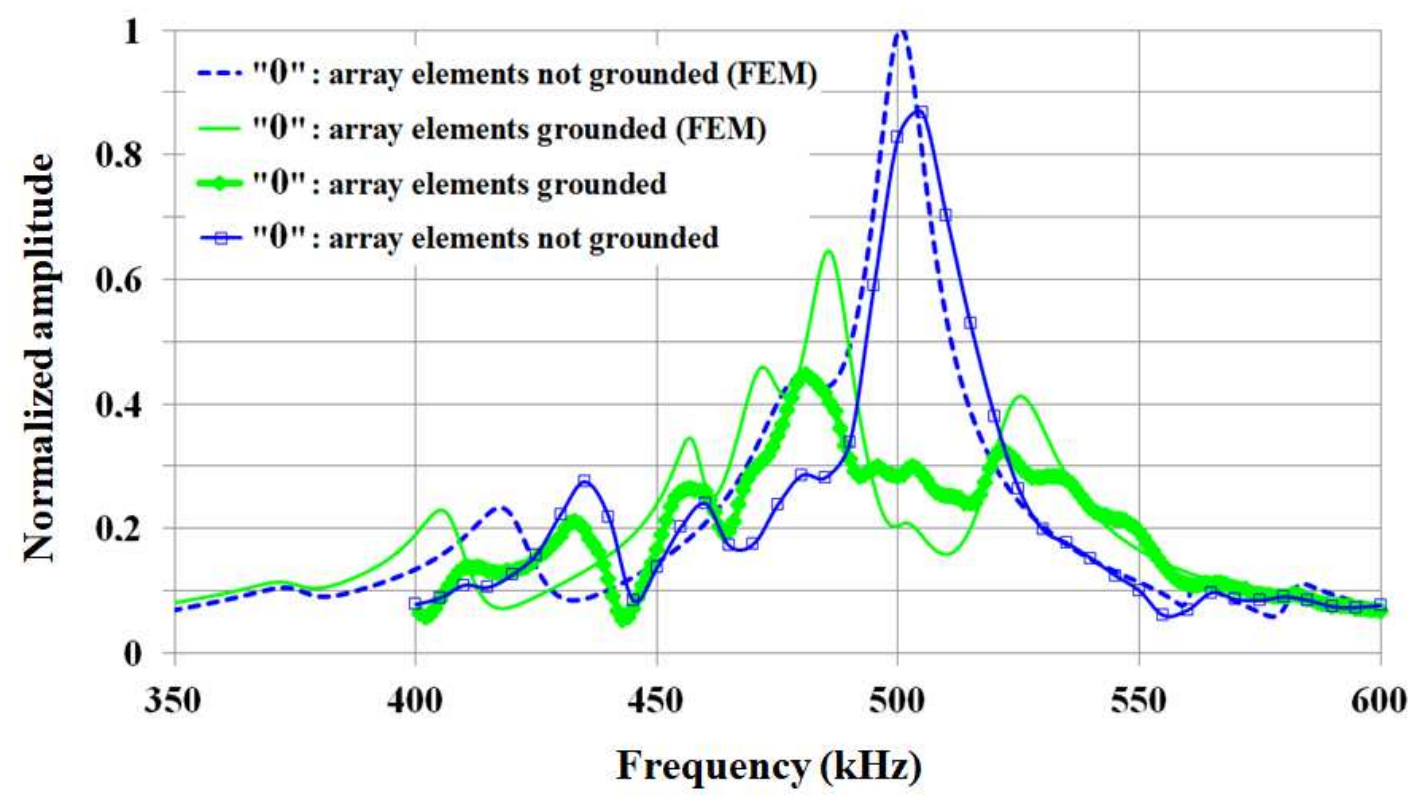

Figure 12: Normalized displacements amplitude measured in the middle of the central element "0" compared to the numerical results (FEM).

\subsection{Electrical impedance}

The electrical impedance of the transducer array is also measured in the frequency band $200 \mathrm{kHz}-800 \mathrm{kHz}$. Fig. 13 compares the curves obtained considering the limit conditions (i) and (ii). To show the effects of the interactions between the array elements (crosstalk), the transducer array's curves are also compared to that of a single piezoelectric element. After analysis, different conclusions can be made. Firstly, concerning the single element, a minimum and a maximum of impedance are observed at $452 \mathrm{kHz}$ and $558 \mathrm{kHz}$ respectively. Secondly, a frequency shift can be observed between the transducer array curves and the 
electrical impedance of the single element (about $30 \mathrm{kHz}$ ). This result is due to the presence of the neighboring piezoelectric elements, which shifts the resonance and anti-resonance frequencies towards the high frequencies. Furthermore, due to the crosstalk phenomenon, parasitic vibrations are obtained around the array's resonance (condition (i)) and antiresonance frequencies (condition (ii)), contrary to the single piezoelectric element. Finally, in the same manner as the numerical results, differences are observed between the electrical impedance curves obtained in the case of the conditions (i) and (ii). Indeed, two resonance frequencies $(481 \mathrm{kHz}$ and $520 \mathrm{kHz})$ and a one anti-resonance frequency $(580 \mathrm{kHz})$ are obtained in the case of the condition (i), whereas in the case of the condition (ii), a one resonance frequency is obtained at $500 \mathrm{kHz}$ and two anti-resonance frequencies are observed at $565 \mathrm{kHz}$ and $583 \mathrm{kHz}$. As seen in Fig. 11, the resonance frequencies (minimum of impedance) correspond also to the mechanical resonance frequencies (maximum of displacement).

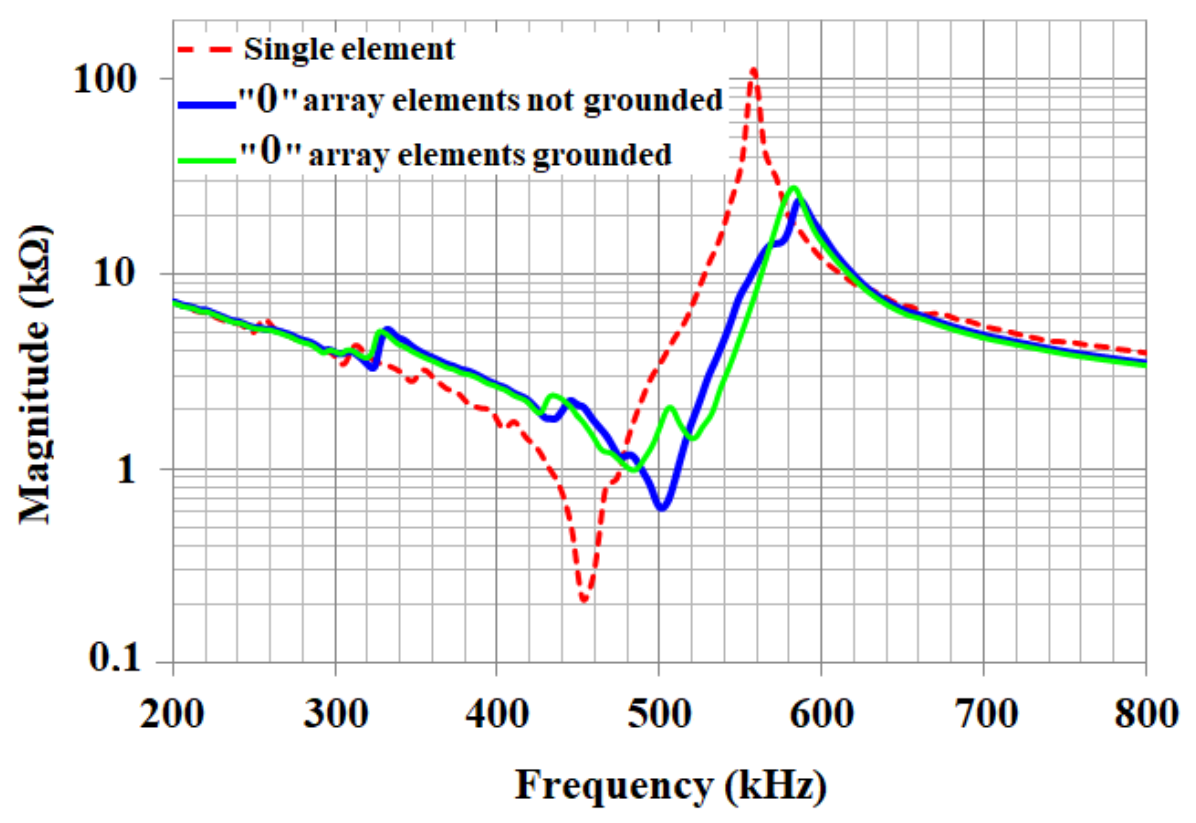

Figure 13: Experimental electrical impedance of the element " 0 " compared to that of a single piezoelectric element (dashed line).

Fig. 14 compares the experimental and numerical (FEM) electrical impedances in both conditions (i) and (ii): array elements grounded and not grounded. It is seen from this figure that the results obtained are similar. Nevertheless, a small frequency shift is observed between the curves in the frequency band $250 \mathrm{kHz}-450 \mathrm{kHz}$, due to the materials' incertitude. Furthermore, in the case of the condition (ii), i.e. when the central element " 1 " is excited and its neighboring elements are grounded, only two anti-resonance frequencies are observed in 
the measured curve, instead of three frequencies as obtained numerically. This result is mainly due to the resin losses chosen in the numerical model (about $5 \%$ ) and the piezoelectric material losses, which are not taken into account.

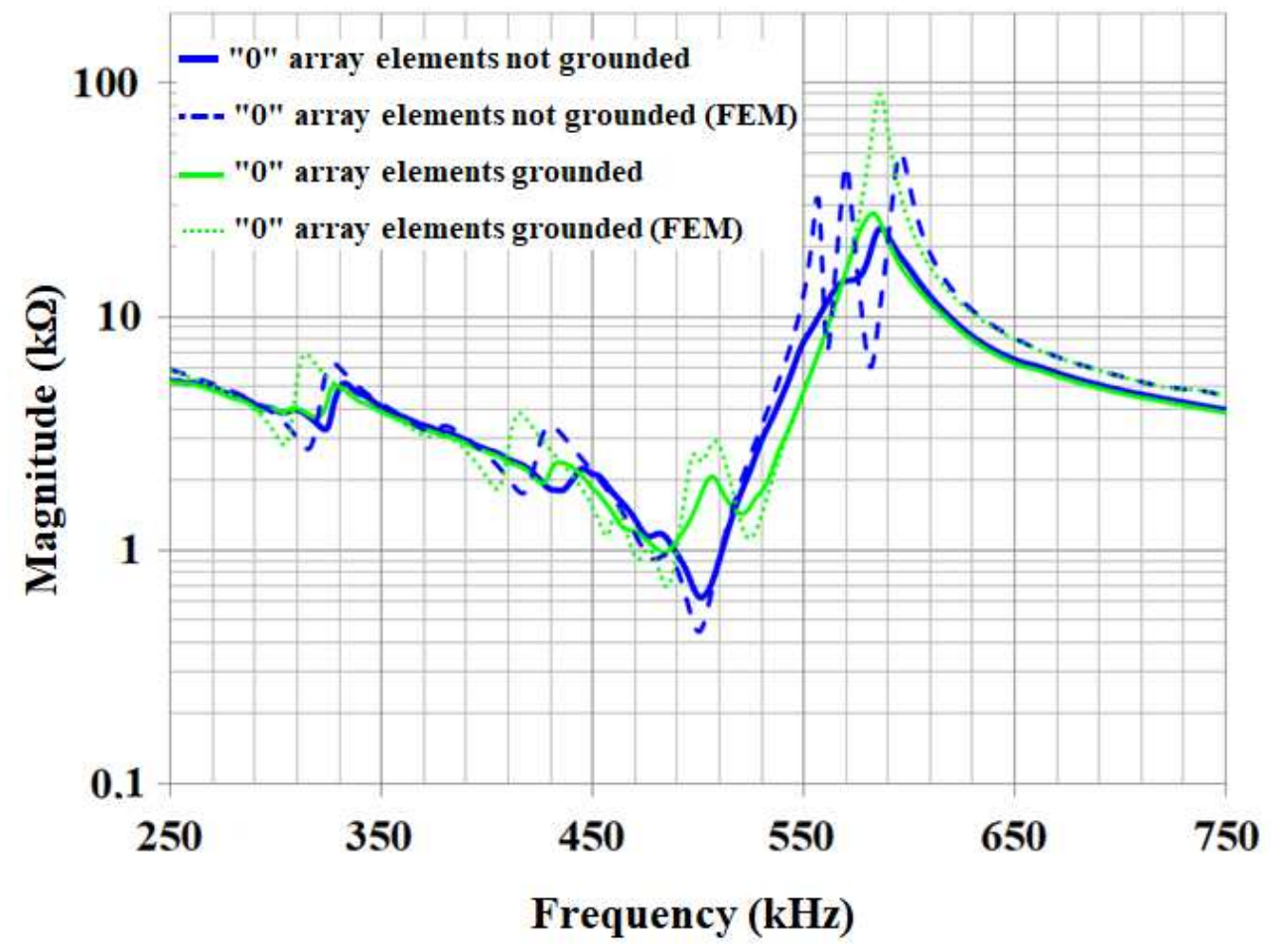

Figure 14: Electrical impedance measured on the element " 0 " compared to the numerical one.

\subsection{Crosstalk}

The experimental setup presented previously in Fig. 3 is first utilized to measure the parasitic voltages generated on the neighboring elements "1", “-1”, “2”, “-2”, “3”, and "-3”, when the central element " 0 " is excited by a harmonic signal having $10 \mathrm{~V}$ amplitude. In this case, measurements are realized at the first resonant frequency of $481 \mathrm{kHz}$. The crosstalk level $C(\mathrm{~dB})$ is then evaluated using the relation (1). The obtained results are given in Table III.

\begin{tabular}{|c|c|c|c|c|c|c|c|}
\hline Element & “-3" & “-2" & “-1" & “0” & “1” & “2” & “3” \\
\hline $\begin{array}{c}\text { Voltage } \\
\text { (V) }\end{array}$ & 5.04 & 1.64 & 3.23 & 10 & 2.73 & 1.62 & 4.47 \\
\hline $\begin{array}{c}\text { Crosstalk } \\
\text { C(dB) }\end{array}$ & -5.95 & -15.7 & -9.81 & 0 & -11.28 & -15.81 & -7 \\
\hline
\end{tabular}

Table III: Crosstalk evaluated using parasitic voltages. 
The measurements show that when the central element " 0 " is excited, it vibrates mainly in its thickness mode, but it generates significant undesirable voltages on its passive neighboring elements "1", “-1", “2”, “-2", “3” and "-3". These results demonstrate the presence of crosstalk in the fabricated transducer array, i.e. the measured average values about $-10.54 \mathrm{~dB},-15.75 \mathrm{~dB}$, and $-6.47 \mathrm{~dB}$ on the first, second and third neighboring elements respectively. Furthermore, a high level of crosstalk is noticed in the case of the third neighboring elements " 3 " and "-3" due to edge effects. As explained previously, in the case of active cancellation of crosstalk, the determination of the correction voltages considers the neighboring elements electrically in Short-Circuit (condition (i)). In this situation, the crosstalk cannot be estimated using the conventional definition (relation (1)). The proposed solution to evaluate the crosstalk level is to measure the parasitic displacements generated on the neighboring elements using the experimental setup given in Fig. 4 and then deduce the crosstalk $C_{u}(\mathrm{~dB})$ from the relation (2). In this case, the results obtained when the central element " 0 " is excited (at the frequency $481 \mathrm{kHz}$ ) and its neighboring elements are connected to the ground (Fig. 4) are summarized in Table IV. In the same manner as the previous results (Table III), significant parasitic displacements are observed on the passive neighboring elements. Consequently, strong crosstalk is noticed on the first (“1”, “-1") and third (“3”, “3") neighboring elements particularly.

\begin{tabular}{|c|c|c|c|c|c|c|c|}
\hline Element & “-3" & “-2" & “-1" & “0” & "1" & “2” & “3” \\
\hline $\begin{array}{c}\text { Displacement } \\
(\mathbf{n m})\end{array}$ & 6.21 & 2.73 & 5.48 & 11.8 & 3.62 & 1.8 & 5.59 \\
\hline $\begin{array}{c}\text { Crosstalk } \\
\boldsymbol{C}_{\boldsymbol{u}}(\mathbf{d B})\end{array}$ & -5.58 & -12.71 & -6.66 & 0 & -10.26 & -16.33 & -6.49 \\
\hline
\end{tabular}

Table IV: Crosstalk evaluated using parasitic displacements.

The comparison of the results given in Tables III and IV shows that the two methods, i.e. estimation of the crosstalk level by the relation (1) and using parasitic displacements instead of voltages, give relatively similar crosstalk values at the resonant frequency. The difference is because the definition of the crosstalk is based on the voltage, which is an average value, whereas, the displacement's method is punctual, i.e. measurements are realized only in the middle of the transducer array elements (Fig. 4), to avoid edge effects. In other words, this method supposes the displacement uniform at the surface of the individual elements, which is not true. For more precision, it would be better to measure the displacement at the whole surface of the array elements, to take into account the contribution 
of the parasitic length mode $[8,16]$. The latter makes the displacement not uniform. Measurements can be achieved with a Scanning Laser Vibrometer, e.g. Polytech psv400. In this situation, average displacement values can be obtained and the precision of crosstalk evaluation can be improved.

To estimate the crosstalk level in a relatively large frequency band $(460 \mathrm{kHz}-520 \mathrm{kHz})$ using the relation (2), displacement measurements are first done in the middle of the array elements. The results obtained on the elements " 0 ", “-1", “-2", and “-3", when the central element " 0 " is excited by a harmonic signal having $1 \mathrm{~V}$ amplitude and its neighboring elements are grounded (limit condition (i)) are shown in Fig. 15. After analysis, it is observed that significant parasitic displacements are obtained on the passive neighboring elements due to the crosstalk phenomenon. At the resonant frequency $481 \mathrm{kHz}$, a maximum of displacement is measured on the central element " 0 " $\left(u_{0}=1.19 \mathrm{~nm}\right)$. The parasitic displacements measured on the element "-1", “-2" and "-3" are about $46 \%, 25 \%$ and $56 \%$ the amplitude of the excited element " 0 " respectively.

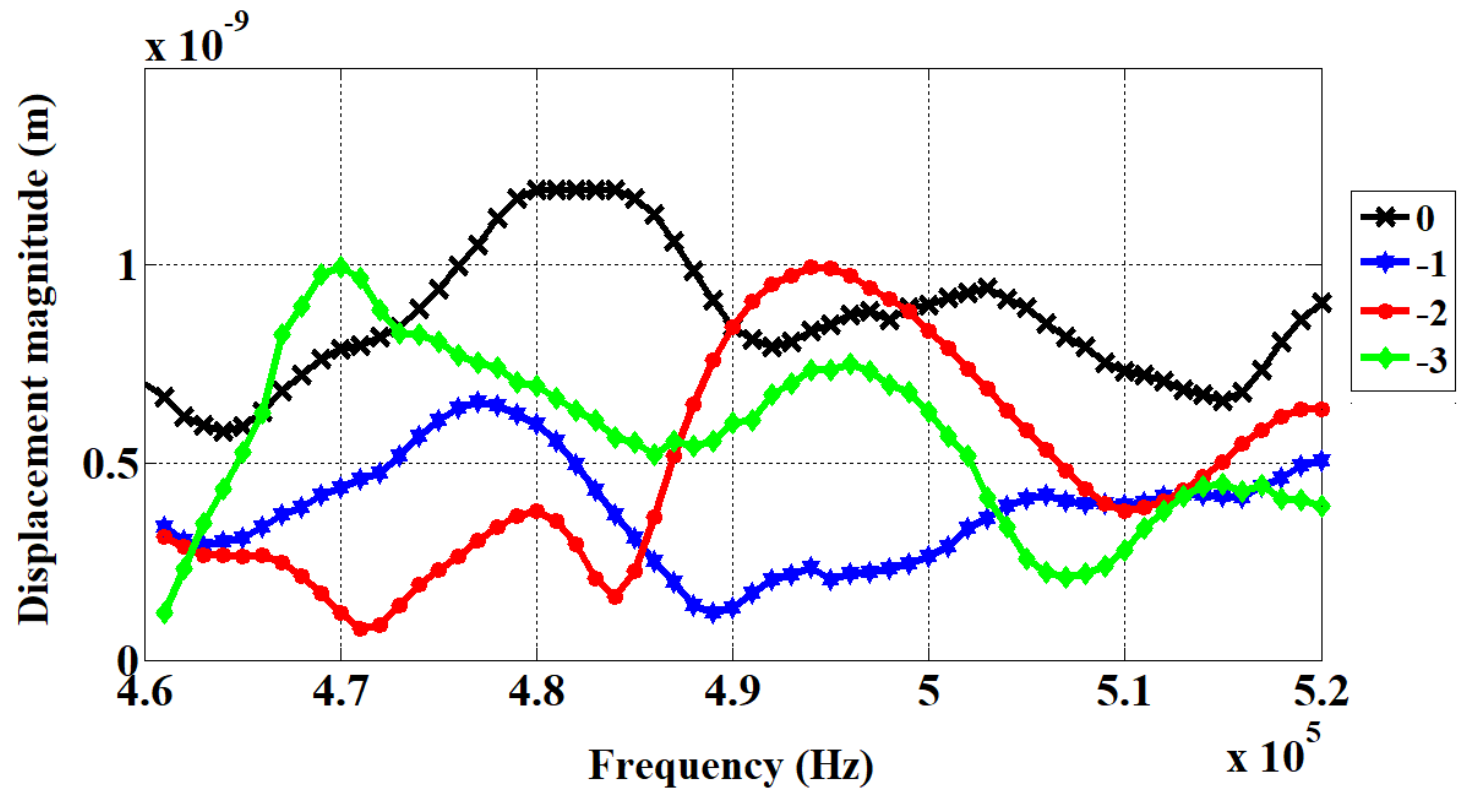

Figure 15: Displacement measured in the middle of the array elements "0", “-1", "-2" and "3 ", when the central element " 0 " is excited by a harmonic signal having $1 \mathrm{~V}$ amplitude and its neighboring elements grounded.

The relation (2) is then utilized to evaluate the crosstalk level in terms of displacement as shown in Fig. 16. According to the latter, it is clear that in the frequency band $460 \mathrm{kHz}-$ $520 \mathrm{kHz}$, the transducer array presents a strong crosstalk level (greater than $-20 \mathrm{~dB}$ ). Around the central element's resonant frequency $481 \mathrm{kHz}$, i.e. between $475 \mathrm{kHz}-487 \mathrm{kHz}$, the 
highest crosstalk level is obtained for the third element "-3", located on the array's edge. In the same frequency band, a relatively similar crosstalk level is measured on the first "-1". Finally, the crosstalk level observed of the second neighbor "-2" is also not negligible (higher than $-20 \mathrm{~dB}$ ). Because of these high crosstalk levels, active crosstalk cancellation methods are successfully proposed and utilized to reduce the interactions between the array elements (crosstalk) and to improve its performance [16, 27].

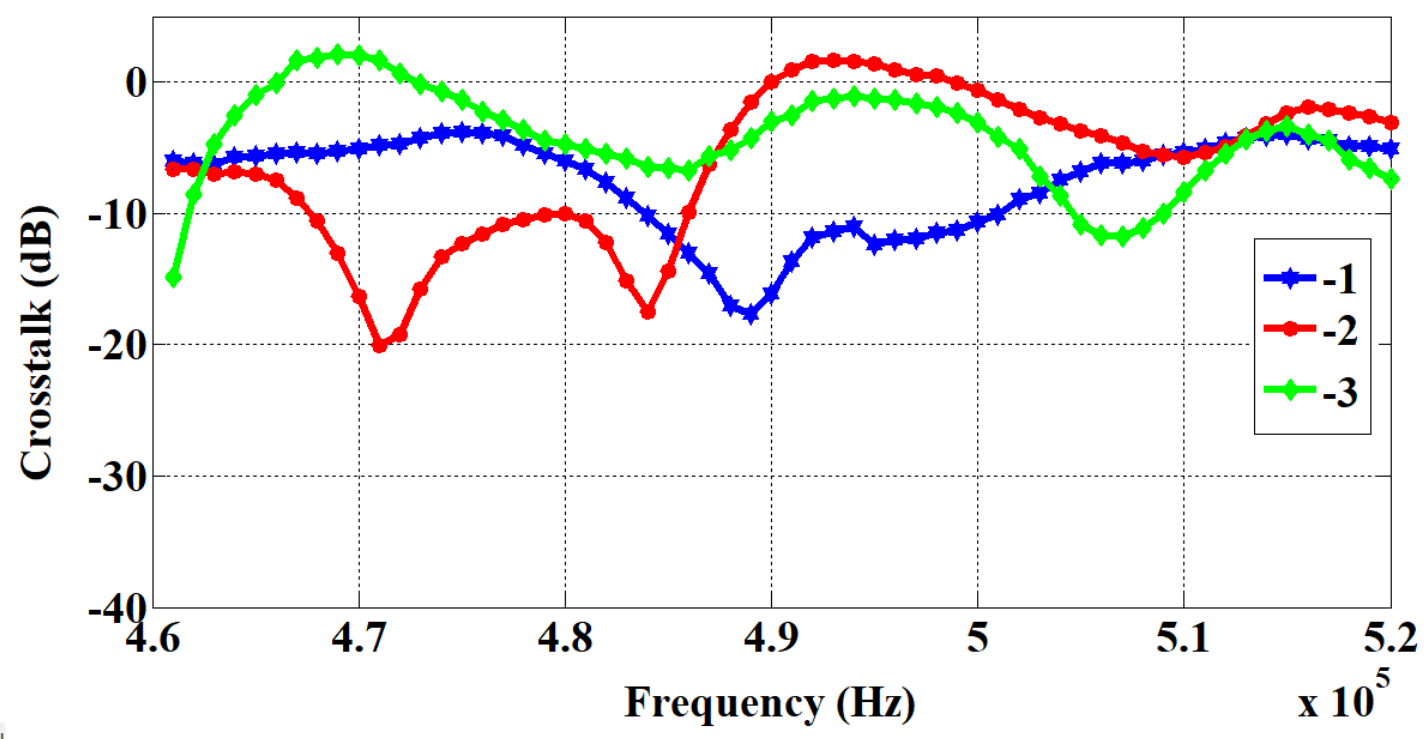

Figure 16: Crosstalk estimated on the array elements "-1", “-2" and "-3" by using the relation 2 (array elements grounded).

\section{Conclusion}

In this paper, the effects of the electrical limit conditions on the electromechanical behavior of a piezoelectric transducer array are investigated numerically and experimentally. A seven-element transducer array radiating in water is first modeled using the 2D Finite Element Method. Globally, the computed results (TVR, electrical impedance and displacement) indicated that the influence of the limit conditions (central element's neighboring elements grounded or not) is mainly observed around the thickness mode's resonance and anti-resonance frequencies. Furthermore, a frequency shift is obtained between the curves computed considering the two limit conditions (about $20 \mathrm{kHz}$ ). However, concerning the crosstalk, it is shown that the values calculated under the two electrical limit conditions are relatively similar (about $1 \mathrm{~dB}$ of difference) at the mechanical resonance frequencies (maximum of displacement) $475 \mathrm{kHz}$ and $520 \mathrm{kHz}$. The difference between the computed curves increases beyond the resonance frequencies. The numerical results 
(electrical impedance and displacements) computed in the air medium are successfully compared to those measured using an impedance analyzer and a Laser Vibrometer respectively. In both cases, it is shown that the influence of the limit conditions is mainly observed around the thickness mode's resonance and anti-resonance frequencies. Furthermore, the crosstalk level is evaluated experimentally at the mechanical resonance frequency $481 \mathrm{kHz}$ using two methods, i.e. voltage and displacement measurements. The results obtained are relatively similar. Nevertheless, for more precision, a Scanning Laser Vibrometer should be utilized to achieve average displacement values instead of punctual ones. Finally, the crosstalk level is estimated in the frequency band $460 \mathrm{kHz}-520 \mathrm{kHz}$ using the proposed method, i.e. deduced from punctual displacement measurement. According to the results, the manufactured seven-element transducer array presents a strong crosstalk level (greater than $-20 \mathrm{~dB}$ ), which should be reduced to improve the array's electromechanical behavior. For this purpose, active crosstalk cancellation methods can be utilized, i.e. the application of adequate correction voltages to the array elements can be a solution to reduce the crosstalk level. Dicing the inter-element filling material can also contribute to reduction of this undesirable phenomenon (crosstalk).

\section{References}

[1] W. Lee and Y. Roh, "Ultrasonic transducers for medical diagnostic imaging," Biomedical Engineering Letters, 7(2):91-97, 2017.

[2] B. Cox, and P. Beard, "Imaging Techniques: Super-Resolution Ultrasound," Nature, 527(7579):451-452, 2015.

[3] X. Jiang, S. Li, J. Kim, J. Ma, W. Huang, and X. Jian, "High Frequency Piezo-Composite Micromachined Ultrasound Transducer Array Technology for Biomedical Imaging," ASME Biomedical and Nanomedical Technologies Concise Monograph Series, ASME Press, New York, 2017.

[4] P. Anastasiadis, A. Mohammadabadi, M. J. Fishman, J. A. Smith, B. A. Nguyen, D. S. Hersh, and V. Frenkel, "Design, characterization and evaluation of a laser-guided focused ultrasound system for preclinical investigations," BioMedical Engineering OnLine, 18(1):36, 2019.

[5] D. S. Hersh, P. Anastasiadis, A. Mohammadabadi, B. A. Nguyen, S. Guo, J. A. Winkles, A. J. Kim, R. Gullapalli, A. Keller, V. Frenkel, and G. F. Woodworth, "MR-guided transcranial focused ultrasound safely enhances interstitial dispersion of large polymeric nanoparticles in the living brain," PLOS ONE, 13(2):e0192240, 2018.

[6] Y. Roh and M. S. Afzal, "Optimal design of a sparse planar array transducer for underwater vehicles by inclusion of crosstalk effect," Japanese Journal of Applied Physics, 57(7S1):07LG02-1-07LG02-7, 2018. 
[7] A. Bybi, O. Mouhat, M. Garoum, H. Drissi, and S. Grondel, "One-dimensional equivalent circuit for ultrasonic transducer arrays," Applied Acoustics, 156:246-257, 2019.

[8] A. Bybi, D. Khouili, C. Granger, M. Garoum, A. Mzerd, and A.-C. Hladky-Hennion, "Experimental Characterization of A Piezoelectric Transducer Array Taking into Account Crosstalk Phenomenon," International Journal of Engineering and Technology Innovation, 10(1):01-14, 2020.

[9] M. Celmer, K. J. Opielinski, and M. Dopierała, "Structural model of standard ultrasonic transducer array developed for FEM analysis of mechanical crosstalk," Ultrasonics, 83:114-119, 2018.

[10] J. Henneberg, A. Gerlach, H. Storck, H. Cebulla, and S. Marburg, "Reducing mechanical cross-coupling in phased array transducers using stop band material as backing," Journal of Sound and Vibration, 424:352-364, 2018.

[11] K. J. Opielinski, M. Celmer, and R. Bolejko, "Crosstalk effect in medical ultrasound tomography imaging," In Joint Conference-Acoustics, pp. 1-6, 2018.

[12] S. Pyo and Y. Roh, "Analysis of the crosstalk in an underwater planar array transducer by the equivalent circuit method," Japanese Journal of Applied Physics, 56(7S1):07JG01-1-07JG01-6, 2017.

[13] W. Lee and Y. Roh, "Optimal design of a piezoelectric 2D array transducer to minimize the cross-talk between active elements," In IEEE Ultrasonics Symposium (IUS), pp. 2738-2741, 2009.

[14] I. S. Domínguez, P. A. Contla, E. M. Hernández, and M. Antônio von Krüger, "Crosstalk effects caused by the geometry of piezoelectric elements in matrix ultrasonic transducers,” Revista Brasileira de Engenharia Biomédica, 27(2):90-97, 2011.

[15] J. Assaad and C. Bruneel, "Radiation from finite phased and focused linear array including interaction," The Journal of the Acoustical Society of America, 101(4):18591867, 1997.

[16] A. Bybi, S. Grondel, A. Mzerd, C. Granger, M. Garoum, and J. Assaad, "Investigation of cross-coupling in piezoelectric transducer arrays and correction," International Journal of Engineering and Technology Innovation, 9(4):287-301, 2019.

[17] F. P. Branca, F. Bini, F. Marinozzi, and A. Grandoni, "Optimum choice of acoustic properties of filling materials using optical measurement," In IEEE Ultrasonics Symposium (IUS), pp. 1663-1665, 2004.

[18] W. Lee and Y. Roh, "Optimal design of a piezoelectric 2D array transducer to minimize the cross-talk between active elements," In IEEE Ultrasonics Symposium (IUS), pp. 2738-2741, 2009.

[19] H. J. Fang, Y. Chen, C. M. Wong, W. B. Qiu, H. L. Chan, J. Y. Dai, Q. Li, and Q. F. Yan, "Anodic aluminum oxide-epoxy composite acoustic matching layers for ultrasonic transducer application," Ultrasonics, 70:29-33, 2016.

[20] M. H. Amini, T. W. Coyle, and T. Sinclair, "Porous ceramics as backing element for high-temperature transducers," IEEE Transactions on Ultrasonics, Ferroelectrics, and Frequency Control, 62(2):360-372, 2015. 
[21] S. M. Ji, J. H. Sung, C. Y. Park, and J. S. Jeong, "Phase-canceled backing structure for lightweight ultrasonic transducer," Sensors and Actuators A: Physical, 260:161-168, 2017.

[22] K. C. T. Nguyen, L. H. Le, M. D. Sacchi, L. Q. Huynh, and E. Lou, "Adaptive noise cancellation in the intercept times-slowness domain for eliminating ultrasonic crosstalk in a transducer array," In Proc. IFMBE, 5th International Conference on Biomedical Engineering in Vietnam, Springer, vol. 46, pp. 32-35, 2015.

[23] C. Ishihara, T. Ikedaa, and H. Masuzawaa, "Higher-frame-rate ultrasound imaging with reduced cross-talk by combining a synthetic aperture and spatial coded excitation," In Proc. SPIE, Medical Imaging, Ultrasonic Imaging and Tomography, vol. 9790, pp. 97901Z-1-97901Z-7, 2016.

[24] B. Cugnet, A.-C. Hladky, and J. Assaad, "Numerical technique to reduce crosscoupling in acoustical arrays," Ultrasonics, 40:503-506, 2002.

[25] S. Zhou, G. L. Wojcik, and J. A. Hossack, "An approach for reducing adjacent element crosstalk in ultrasound arrays," IEEE Trans. Ultrason. Ferroelectr. Freq. Contr., 50(12):1752-1761, 2003.

[26] S. Zhou, G. L. Wojcik, and J. A. Hossack, "Reducing inter-element acoustic crosstalk in capacitive micromachined ultrasound transducers," IEEE Trans. Ultrason. Ferroelectr. Freq. Contr., 54(6):1217-1228, 2007.

[27] A. Bybi, C. Granger, S. Grondel, A. C. Hladky -Hennion, and J. Assaad, "Electrical method for crosstalk cancellation in transducer arrays," NDT \& E International, 62:115$121,2014$.

[28] ATILA, Finite-Element Software Package for the analysis of 2D \& 3D structures based on smart materials Version 6.0.2 User's Manual, November (2010).

[29] J. Sato, M. Kawabuchi, and A. Fukumoto, "Dependence of electromechanical coupling coefficient on the width-to-thickness ratio plank-shaped piezoelectric transducers used for electronically scanned ultrasound diagnostic systems," The Journal of the Acoustical Society of America, 66(6):1609-1611, 1979.

[30] W. Friedrich, H. Kaarmann, and R. Lerch, "Finite element modeling of acoustic radiation from piezoelectric phased antennas," In IEEE Ultrasonics Symposium (IUS), pp. 763767, 1990. 